\title{
Scale-invariant diffusions: transience and non-polar points
}

\author{
RICHARD DANTE DEBLASSIE \\ Department of Mathematics, Texas A\&M University, College Station, TX 77843, USA. \\ E-mail: deblass@math.tamu.edu
}

Consider a diffusion in $\mathbb{R}^{d}(d \geqslant 2)$ whose generator has coefficients independent of the distance to the origin. Then there is a parameter $\alpha$ so that the origin is almost surely hit when $\alpha<1$ and almost surely not hit when $\alpha>1$. Moreover, the process is transient to $\infty$ for $\alpha>1$. We identify $\alpha$ in terms of the diffusion coefficients and a certain invariant measure. In some special two-dimensional cases we explicitly compute the invariant measure and resolve the critical case $\alpha=1$. This work complements and extends certain results of Pinsky (1995) and Williams (1985).

Keywords: invariant measure; martingale problem; recurrence; scale-invariant diffusions; transience

\section{Introduction}

Bass and Pardoux (1987) study diffusions in $\mathbb{R}^{d}(d \geqslant 2)$ corresponding to uniformly elliptic operators of the form

$$
\frac{1}{2} \sum_{i, j=1}^{d} a_{i j}(x) \frac{\partial^{2}}{\partial x_{i} \partial x_{j}},
$$

where $a_{i j}(x)=a_{i j}(x /|x|), x \neq 0$. Here 'uniformly elliptic' means $\left(a_{i j}(x)\right)$ is a symmetric matrix and, for some $\lambda>0$,

$$
\lambda|\xi|^{2} \leqslant \sum_{i, j=1}^{d} a_{i j}(x) \xi_{i} \xi_{j} \leqslant \frac{1}{\lambda}|\xi|^{2}, \quad x \neq 0, \xi \in \mathbb{R}^{d} .
$$

One of their main results asserts that if the diffusion is uniquely determined up to the first hitting time of the origin, then the process can be uniquely extended to a diffusion for all times. The major difficulty they encounter arises from the intriguing possibility of the process hitting 0 with positive probability. This is due (more or less) to the presence of a discontinuity at 0 in the coefficients $a_{i j}$ (DeBlassie 1998). The authors provide an interesting example in which the origin is hit almost surely. Under some regularity conditions on the $a_{i j}$, among other interesting results, Pinsky (1995) shows that diffusions governed by (1.1) either almost surely hit 0 or almost surely do not hit 0 . There is a parameter whose sign determines this behaviour. Pinsky also discusses transience and recurrence. See Theorem 3.1 and the subsequent remarks in Section 6.3 of that book. 
We take a different approach, reducing assumptions on the coefficients $a_{i j}$ by assuming uniqueness of the associated martingale problem, stopped upon hitting the origin. A situation considered by Bass and Pardoux (piecewise constant coefficients) falls under our assumptions, whereas Pinsky's results do not apply.

The key property is Brownian-like scaling. We consider slightly more general uniformly elliptic operators of the form

$$
L=\frac{1}{2} \sum_{i, j=1}^{d} a_{i j}(x) \frac{\partial^{2}}{\partial x_{i} \partial x_{j}}+\sum_{i=1}^{d} \frac{1}{|x|} b_{i}(x) \frac{\partial}{\partial x_{i}},
$$

where, for each $i, j=1, \ldots, d$,

$$
\begin{array}{ll}
a_{i j}(x)=a_{i j}(x /|x|), & x \neq 0, \\
b_{i}(x)=b_{i}(x /|x|), & x \neq 0,
\end{array}
$$

are bounded and measurable. Let $D \subseteq S^{d-1}$ denote the set of discontinuities of $a$ and $b$ regarded as functions on the unit sphere $S^{d-1}$. Let $\mu$ denote normalized $\left(\mu\left(S^{d-1}\right)=1\right)$ surface measure for $d \geqslant 3$ and arc length for $d=2$, on $S^{d-1}$. We assume

$$
\mu(D)=0 \text {. }
$$

Note that the drift is given the specified form so as to preserve the Brownian-like scaling. Our final assumption is that the martingale problem for $L$ is well posed up to the first hitting time of 0 . The precise meaning of this is given below in Section 2. Denote by $X(t)$ the corresponding diffusion.

Let $(\theta, r)$ be polar coordinates in $\mathbb{R}^{d}: r=r(x)=|x|$ and $\theta=\theta(x)$ is the point on $S^{d-1}$ corresponding to $x /|x|, x \neq 0$. By (1.2) and (1.3), $L$ takes on the form

$$
L=A(\theta)\left[\frac{1}{2} \frac{\partial^{2}}{\partial r^{2}}+\frac{1}{2 r} B(\theta) \frac{\partial}{\partial r}+\frac{1}{r} \frac{\partial}{\partial r} \bar{L}_{1}+\frac{1}{r^{2}} \bar{L}_{2}\right]
$$

where

$$
\begin{aligned}
& A(\theta)=(a(x) x, x) / r^{2}, \\
& B(\theta)=[\operatorname{tr} a(x)+2(x, b(x)) / r] /\left[(a(x) x, x) / r^{2}\right]-1
\end{aligned}
$$

(in which $(\cdot, \cdot)$ is the usual Euclidean inner product), and $\bar{L}_{1}, \bar{L}_{2}$ are first- and second-order differential operators, respectively, on $C^{2}\left(S^{d-1}\right)$.

Below we will show that the diffusion on $S^{d-1}$ corresponding to $\bar{L}_{2}$ is uniquely determined and has a unique invariant probability measure $v$ (see Theorems 2.2 and 3.2). Define

$$
\alpha=\int_{S^{d-1}} B(\theta) v(\mathrm{~d} \theta)
$$

Theorem 1.1. Assume that the martingale problem for $L$ is well posed up to the first hitting time $\tau_{0}$ of 0 , and suppose (1.3)-(1.5) are in effect. Let $X_{0} \neq 0$. 
(i) If $\alpha>1$ then $X$ is transient to $\infty$ and almost surely does not hit 0 .

(ii) If $\alpha<1$ then on the set $\left\{\tau_{0}=\infty\right\}, X_{t} \rightarrow 0$ as $t \rightarrow \infty$.

(iii) If $\alpha<1$ and $\inf _{x \neq 0}[\operatorname{tr} a(x)+2 x \cdot b(x) /|x|]>0$ then $\tau_{0}<\infty$ almost surely.

Remarks. 1. If $\alpha<1$ and $\sup _{x \neq 0}[\operatorname{tr} a(x)+2 x \cdot b(x) /|x|]<0$, then the conclusion of (iii) holds. The proof is similar.

2. If $b \equiv 0$, then we are in the case Bass and Pardoux consider. By strict ellipticity, inf $\operatorname{tr} a>0$, and so the hypothesis about the infimum in (iii) holds automatically. Thus in this case, the process either almost surely does not hit $0(\alpha>1)$ or almost surely does hit 0 $(\alpha<1)$.

3. We are able to resolve the critical case $\alpha=1$ only in special circumstances. See Section 5 below.

The greatest source of difficulty is evident from the form of $L$ in (1.6): there is highly non-trivial interaction between the radial and angular parts arising from the $B(\theta)$ and $\bar{L}_{1}$ terms. Because of this, there is no skew product representation of the diffusion. With such a representation, our results would be easy to derive.

The rest of our results are concerned with two-dimensional cases in which we are able to identify $\alpha$ in explicit form and discuss the critical case $\alpha=1$. See Theorems 4.1 and 5.1 below.

A special case is covered by some work in Williams (1985). She studies Brownian motion with polar drift, where $L$ takes the form

$$
\frac{1}{2} \Delta+\frac{\gamma(\theta)}{2 r} \frac{\partial}{\partial r}
$$

with $\gamma(\theta)$ bounded and measurable. She shows

$$
\alpha=\int_{S^{d-1}} \gamma(\theta) \mathrm{d} \mu(\theta)+d-1
$$

Moreover, when $\alpha=1$, the process almost surely does not hit 0 (starting away from 0 ) and is finely recurrent. Here the facts that $\bar{L}_{1}$ is absent and $\bar{L}_{2}$ is more or less the Laplacian on $S^{d-1}$ are crucial to obtaining these results. Pinsky (1995) also resolves the critical case $\alpha=1$ under further regularity hypotheses on the coefficients.

The paper is organized as follows. The proof of Theorem 1.1 is given in Section 2, assuming the existence of a unique invariant probability measure for a time change of the angular part of $X$. The proof of the latter is the content of Section 3. In the course of the proof, we show (roughly speaking) that uniqueness of the stopped martingale problem for $L$ implies uniqueness of the martingale problem for the angular part, a result of independent interest. In Section 4 we explicitly identify the invariant probability measure for $d=2$ in the case of piecewise smooth coefficients. Finally, in Section 5 we study the critical case $\alpha=1$ for piecewise constant coefficients in two dimensions. 


\section{Proof of Theorem 1.1}

Let $\Omega_{d}=C\left([0, \infty), \mathbb{R}^{d}\right)$ and denote the coordinate process by $X_{t}$. Define

$$
\begin{aligned}
\mathscr{F}_{t} & =\sigma\left(X_{s}: 0 \leqslant s \leqslant t\right), \\
\mathscr{F} & =\sigma\left(X_{s}: 0 \leqslant s<\infty\right), \\
\tau_{0} & =\inf \left\{\mathrm{t} \geqslant 0: X_{t}=0\right\} .
\end{aligned}
$$

Given $x \in \mathbb{R}^{d} \backslash\{0\}$, a solution to the $\tau_{0}$-stopped martingale problem for $L$ is a probability measure $P_{x}$ on $\left(\Omega_{d}, \mathscr{F}\right)$ such that

$$
P_{0}\left(X_{0}=0\right)=1
$$

for each $f \in C_{0}^{2}\left(\mathbb{R}^{2}\right)$,

$$
f\left(X\left(t \wedge \tau_{0}\right)\right)-\int_{0}^{t \wedge \tau_{0}}(L f)\left(X_{s}\right) \mathrm{d} s
$$

is a $P_{x}$-martingale;

$$
P_{x}\left(X_{t}=0 \text { for } t \geqslant \tau_{0}\right)=1 .
$$

We call $X(\cdot)$ under $P_{x}$ the process absorbed at 0 , or simply the absorbed process. As stated above, throughout this paper we assume $P_{x}$ is uniquely determined for each $x \neq 0$. Let $P_{x}^{0}$ be the law of $X(\cdot)$ under $P_{x}$ killed upon first hitting 0 :

$$
P_{x}^{0}\left(\bigcap_{i=1}^{m}\left\{X\left(t_{i}\right) \in B_{i}\right\}\right)=P_{x}\left(\bigcap_{i=1}^{m}\left\{X\left(t_{i}\right) \in B_{i}, \tau_{0}>t_{i}\right\}\right) .
$$

Then $P_{x}^{0}$ is uniquely determined.

By uniform ellipticity and a change of time, it is enough to prove the theorem for the operator

$$
\bar{L}=A(\theta)^{-1} L,
$$

where $A(x)=A(x /|x|)$ is from (1.7). We write $\bar{P}_{x}$ and $\bar{P}_{x}^{0}$ for the $\bar{L}$-analogues of $P_{x}$ and $P_{x}^{0}$, respectively. Note that $\bar{P}_{x}^{0}$ is uniquely determined.

Lemma 2.1. Under $\bar{P}_{x}$ with $x \neq 0$,

$$
\int_{0}^{\tau_{0}}\left|X_{s}\right|^{-2} \mathrm{~d} s:=\lim _{t \uparrow \tau_{0}} \int_{0}^{t}\left|X_{s}\right|^{-s} \mathrm{~d} s=\infty \text { almost surely. }
$$

Proof. Let $\sigma: \mathbb{R}^{d} \rightarrow \mathbb{R}^{d} \otimes \mathbb{R}^{d}$ satisfy $\sigma \sigma^{*}=a$. Then under $\bar{P}_{x}$ we can write, for $t<\tau_{0}$,

$$
X_{t}=x+\int_{0}^{t} A\left(X_{s}\right)^{-1 / 2} \sigma\left(X_{s}\right) \mathrm{d} B s+\int_{0}^{t} A\left(X_{s}\right)^{-1}\left[b\left(X_{s}\right) /\left|X_{s}\right|\right] \mathrm{d} s,
$$

where $B$ is a standard $d$-dimensional Brownian motion. By Itô's formula, for $t<\tau_{0}$, 


$$
\left|X_{t}\right|^{2}=|x|^{2}+\int_{0}^{t} 2 A\left(X_{s}\right)^{-1 / 2}\left(X_{s}, \sigma\left(X_{s}\right) \mathrm{d} B_{s}\right)+\int_{0}^{t} A\left(X_{s}\right)^{-1}\left[\operatorname{tr} a\left(X_{S}\right)+2\left(X_{s}, b\left(X_{s}\right)\right) /\left|X_{s}\right|\right] \mathrm{d} s .
$$

Define

$$
F(x)= \begin{cases}(a(x) x, x)^{-1 / 2}, & x \neq 0 \\ 1, & x=0\end{cases}
$$

and set, for $t<\tau_{0}$,

$$
\beta_{t}=\int_{0}^{t} F\left(X_{s}\right)\left(X_{s}, \sigma\left(X_{s}\right) \mathrm{d} B_{s}\right)
$$

Then for $t<\tau_{0}$ the quadratic variation of $\beta$ is

$$
\langle\beta\rangle_{t}=\int_{0}^{t} F\left(X_{s}\right)^{2}\left(a\left(X_{s}\right) X_{s}, X_{s}\right) \mathrm{d} s=t .
$$

Thus up to time $\tau_{0}, \beta_{t}$ is one-dimensional Brownian motion and (2.6) can be rewritten as

$$
\left|X_{t}\right|^{2}=|x|^{2}+\int_{0}^{t} 2\left|X_{s}\right| \mathrm{d} \beta_{s}+\int_{0}^{t} A\left(X_{s}\right)^{-1}\left[\operatorname{tr} a\left(X_{s}\right)+G\left(X_{s}\right)\right] \mathrm{d} s
$$

for $t<\tau_{0}$, where $G(x)=2(x, b(x)) /|x|, x \neq 0$.

To analyse this, let $x_{t}$ be the square of a Bessel process with parameter $\lambda=\sup _{x} A(x)^{-1}$ $[\operatorname{tr} a(x)+G(x)]<\infty:$

$$
x_{t}=|x|^{2}+\int_{0}^{t} 2 \sqrt{x_{s} \vee 0} \mathrm{~d} \beta_{s}+\lambda t
$$

(See example 8.3 of Ikeda and Watanabe 1989, p. 237). Here we extend $\beta$ to times $t \geqslant \tau_{0}$ in the obvious way. Then by a comparison theorem (Theorem 1.1 of Ikeda and Watanabe 1989, p. 437),

$$
\left|X_{t}\right|^{2} \leqslant x_{t}, \quad t<\tau_{0}(X) \text { a.s. }
$$

In particular, on the set $\left\{\tau_{0}(X)=\infty\right\}$,

$$
\begin{aligned}
\int_{0}^{\tau_{0}(X)}\left|X_{s}\right|^{-2} \mathrm{~d} s & =\lim _{t \uparrow \tau_{0}(X)} \int_{0}^{t}\left|X_{s}\right|^{-2} \mathrm{~d} s \\
& =\lim _{t \uparrow \infty} \int_{0}^{t}\left|X_{s}\right|^{-2} \mathrm{~d} s \\
& \geqslant \lim _{t \uparrow \infty} \int_{0}^{t} x_{s}^{-1} \mathrm{~d} s \\
& =\infty,
\end{aligned}
$$

(see DeBlassie 1988) as desired.

To handle the set $\left\{\tau_{0}(X)<\infty\right\}$, note that, by Itô's formula and (2.7), for $t<\tau_{0}(X)$, 
$\log \left|X_{t}\right|^{2}=\log |x|^{2}+\int_{0}^{t} 2\left|X_{S}\right|^{-1} \mathrm{~d} \beta_{s}+\int_{0}^{t}\left|X_{S}\right|^{-2}\left[A\left(X_{S}\right)^{-1}\left\{\operatorname{tr} a\left(X_{S}\right)+G\left(X_{S}\right)\right\}-2\right] \mathrm{d} s$.

Then (see Theorem 7.2' of Ikeda and Watanabe 1989, p. 91), on an extension of $\left(\Omega_{d}, \mathscr{F}, \bar{P}_{x}\right)$, there is a one-dimensional Brownian motion $\tilde{\beta}$ such that, for $t<\tau_{0}(X)$,

$\log \left|X_{t}\right|^{2}=\log |x|^{2}+\tilde{\beta}\left(\int_{0}^{t} 4\left|X_{s}\right|^{-2} \mathrm{~d} s\right)+\int_{0}^{t}\left|X_{s}\right|^{-2}\left[A\left(X_{s}\right)^{-1}\left\{\operatorname{tr} a\left(X_{s}\right)+G\left(X_{s}\right)\right\}-2\right] \mathrm{d} s$.

Hence substituting $\tau_{\delta}=\inf \left\{t \geqslant 0:\left|X_{t}\right|=\delta\right\}$ for $t$, we have, on $\left\{\tau_{0}(X)<\infty\right\}$,

$$
\begin{aligned}
2 \log \delta= & \log \left|X_{\tau_{\delta}}\right|^{2}=\log |x|^{2}+\tilde{\beta}\left(\int_{0}^{\tau_{\delta}} 4\left|X_{S}\right|^{-2} \mathrm{~d} s\right) \\
& +\int_{0}^{\tau_{\delta}}\left|X_{S}\right|^{-2}\left[A\left(X_{S}\right)^{-1}\left\{\operatorname{tr} a\left(X_{s}\right)+G\left(X_{S}\right)\right\}-2\right] \mathrm{d} s .
\end{aligned}
$$

If $\int_{0}^{\tau_{0}}\left|X_{s}\right|^{-2} \mathrm{~d} s<\infty$, then as $\delta \rightarrow 0$ both

$$
\begin{aligned}
& \tilde{\beta}\left(\int_{0}^{\tau_{\delta}} 4\left|X_{S}\right|^{-2} \mathrm{~d} s\right) \text { and } \\
& \int_{0}^{\tau_{\delta}}\left|X_{S}\right|^{-2}\left[A\left(X_{S}\right)^{-1}\left\{\operatorname{tr} a\left(X_{S}\right)+G\left(X_{S}\right)\right\}-2\right] \mathrm{d} s
\end{aligned}
$$

converge a.s. On the other hand, $\log \delta \rightarrow-\infty$ as $\delta \rightarrow 0$, giving a contradiction in (2.10). Hence $\int_{0}^{\tau_{0}}\left|X_{s}\right|^{-2} \mathrm{~d} s=\infty$ on $\left\{\tau_{0}<\infty\right\}$, as claimed.

Now we use the polar coordinates $(\theta, r)$ from Section 1. By Lemma 2.1, the function

$$
\eta_{t}=\int_{0}^{t}\left|X_{s}\right|^{-2} \mathrm{~d} s, t<\tau_{0}
$$

is continuous and increasing with a continuous increasing inverse

$$
\eta_{t}^{-1}:[0, \infty) \rightarrow\left[0, \tau_{0}\right)
$$

Hence the process

$$
\Theta(t)=\theta\left(X\left(\eta_{t}^{-1}\right)\right), \quad t \geqslant 0,
$$

is well defined. The main properties of $\Theta$ we need are contained in the next theorem. Its proof is deferred to Section 3.

Theorem 2.2. The process $\Theta$ has a unique invariant probability measure $v$ on $S^{d-1}$ and the following ergodic result holds:

$$
\lim _{t \rightarrow \infty} \frac{1}{t} \int_{0}^{t} f\left(\Theta_{s}\right) \mathrm{d} s=\int_{S^{d-1}} f(\theta) v(\mathrm{~d} \theta) \text { a.s. }
$$

for any bounded measurable function $f: S^{d-1} \rightarrow \mathbb{R}$. 
Now we complete the proof of Theorem 1.1.

Since $G(x), A(x)$, and $\operatorname{tr} a(x)$ depend only on $x /|x|, x \neq 0$, writing $G(\theta)$ and $A(\theta)^{-1} \operatorname{tr} a(\theta)$ for $\theta \in S^{d-1}$ should cause no confusion. By (2.9) and the definition of $\Theta$,

$$
\begin{aligned}
\log \left|X_{t \wedge \tau_{\delta}}\right|^{2}= & \log |x|^{2}+\tilde{\beta}\left(\int_{0}^{t \wedge \tau_{\delta}} 4\left|X_{s}\right|^{-2} \mathrm{~d} s\right)+\int_{0}^{t \wedge \tau_{\delta}}\left|X_{s}\right|^{-2}\left[A\left(X_{s}\right)^{-1}\left\{\operatorname{tr} a\left(X_{s}\right)+G\left(X_{s}\right)\right\}-2\right] \mathrm{d} s \\
= & \log |x|^{2}+\tilde{\beta}\left(\int_{0}^{t \wedge \tau_{\delta}} 4\left|X_{s}\right|^{-2} \mathrm{~d} s\right) \\
& +\int_{0}^{t \wedge \tau_{\delta}}\left|X_{S}\right|^{-2}\left[A\left(\Theta\left(\eta_{s}\right)\right)^{-1}\left\{\operatorname{tr} a\left(\Theta\left(\eta_{s}\right)\right)+G\left(\Theta\left(\eta_{s}\right)\right)\right\}-2\right] \mathrm{d} s \\
= & \log |x|^{2}+\tilde{\beta}\left(\int_{0}^{t \wedge \tau_{\delta}} 4\left|X_{s}\right|^{-2} \mathrm{~d} s\right)+\int_{0}^{\eta\left(t \wedge \tau_{\delta}\right)}\left[A\left(\Theta_{s}\right)^{-1}\left\{\operatorname{tr} a\left(\Theta_{s}\right)+G\left(\Theta_{s}\right)\right\}-2\right] \mathrm{d} s .
\end{aligned}
$$

On the set $\left\{\tau_{0}=\infty\right\}, \tau_{\delta} \rightarrow \infty$ as $\delta \rightarrow 0$, so (2.11) becomes

$$
\log \left|X_{t}\right|^{2}=\log |x|^{2}+\tilde{\beta}\left(\int_{0}^{t} 4\left|X_{s}\right|^{-2} \mathrm{~d} s\right)+\int_{0}^{\eta(t)}\left[A\left(\Theta_{s}\right)^{-1}\left\{\operatorname{tr} a\left(\Theta_{s}\right)+G\left(\Theta_{s}\right)\right\}-2\right] \mathrm{d} s .
$$

Since $\eta_{t} \rightarrow \infty$ on $\left\{\tau_{0}=\infty\right\}$ as $t \rightarrow \infty$,

$$
\begin{aligned}
\frac{\tilde{\beta}\left(4 \eta_{t}\right)}{\eta_{t}} & \rightarrow 0 \\
\frac{1}{\eta_{t}} \int_{0}^{\eta_{t}}\left[A\left(\Theta_{s}\right)^{-1}\left\{\operatorname{tr} a\left(\Theta_{s}\right)+G\left(\Theta_{s}\right)\right\}-2\right] \mathrm{d} s & \rightarrow \int_{S^{d-1}} A(\theta)^{-1}\{\operatorname{tr} a(\theta)+G(\theta)\} v(\mathrm{~d} \theta)-2 \\
& =\alpha-1 \text { (by (1.7)-(1.9)) }
\end{aligned}
$$

by Theorem 2.2. Hence (recall we never consider $\alpha=1$ )

$$
\lim _{t \rightarrow \infty} \log \left|X_{t}\right|^{2}=\log |x|^{2}+\infty \cdot \operatorname{sign}(\alpha-1), \quad \text { on }\left\{\tau_{0}=\infty\right\} .
$$

First assume $\alpha>1$. By (2.12),

$$
\lim _{t \rightarrow \infty}\left|X_{t}\right|=\infty \quad \text { on }\left\{\tau_{0}=\infty\right\}
$$

On the set $\left\{\tau_{0}<\infty\right\}$, let $t \rightarrow \infty$ in (2.11) to obtain

$$
\begin{aligned}
\log \delta^{2}= & \log \left|X_{\tau_{\delta}}\right|^{2}=\log |x|^{2}+\tilde{\beta}\left(\int_{0}^{\tau_{\delta}} 4\left|X_{s}\right|^{-2} \mathrm{~d} s\right) \\
& +\int_{0}^{\eta\left(\tau_{\delta}\right)}\left[A\left(\Theta_{s}\right)^{-1}\left\{\operatorname{tr} a\left(\Theta_{s}\right)+G\left(\Theta_{s}\right)\right\}-2\right] \mathrm{d} s .
\end{aligned}
$$


Since $\eta\left(\tau_{\delta}\right) \rightarrow \eta\left(\tau_{0}\right)=\infty$ (by Lemma 2.1) as $\delta \rightarrow 0$,

$$
\begin{gathered}
\frac{\tilde{\beta}\left(4 \eta\left(\tau_{\delta}\right)\right)}{\eta\left(\tau_{\delta}\right)} \rightarrow 0 \\
\frac{1}{\eta\left(\tau_{\delta}\right)} \int_{0}^{\eta\left(\tau_{\delta}\right)}\left[A\left(\Theta_{s}\right)^{-1}\left\{\operatorname{tr} a\left(\Theta_{s}\right)+G\left(\Theta_{s}\right)\right\}-2\right] \mathrm{d} s \rightarrow \alpha-1
\end{gathered}
$$

on $\left\{\tau_{0}<\infty\right\}$ as $\delta \rightarrow 0$. Thus if $\bar{P}_{x}\left(\tau_{0}<\infty\right)>0$, then by (2.14)

$$
-\infty=\lim _{\delta \rightarrow 0} \log \left|X_{\tau_{\delta}}\right|^{2}=\log |x|^{2}+\lim _{\delta \rightarrow 0}(\alpha-1) \eta\left(\tau_{\delta}\right)=\infty
$$

with positive probability. Hence $\bar{P}_{x}\left(\tau_{0}<\infty\right)=0$, and combined with (2.13), we obtain part (i) of Theorem 1.1.

Next assume $\alpha<1$. Part (ii) of Theorem 1.1 is an immediate consequence of (2.12). As for part (iii), by (2.12)

$$
\lim _{t \rightarrow \infty}\left|X_{t}\right|^{2}=0 \quad \text { on }\left\{\tau_{0}=\infty\right\}
$$

We use this to show $\bar{P}_{x}\left(\tau_{0}<\infty\right)=1$, giving the desired conclusion. With

$$
c_{1}=\inf _{x} A(x)^{-1}[\operatorname{tr} a(x)+G(x)],
$$

consider the squared Bessel process $\gamma_{t}$ with parameter $c_{1}$ :

$$
\gamma_{t}=|x|^{2}+\int_{0}^{t} 2 \sqrt{\gamma_{s} \vee 0} \mathrm{~d} \beta_{s}+c_{1} t .
$$

By (2.7) and the comparison theorem cited above,

$$
\gamma_{t} \leqslant\left|X_{t}\right|^{2} \text { a.s. }
$$

Hence by (2.15), $\gamma_{t} \rightarrow 0$ on $\left\{\tau_{0}(X)=\infty\right\}$ as $t \rightarrow \infty$. Then if $\bar{P}_{x}\left(\tau_{0}=\infty\right)>0, \gamma_{t} \rightarrow 0$ as $t \rightarrow \infty$ with positive probability. But by hypothesis, $c_{1}>0$ so $\gamma_{t}$ is either recurrent or transient to $\infty$. This contradiction forces $\bar{P}_{x}\left(\tau_{0}=\infty\right)=0$, or equivalently $\bar{P}_{x}\left(\tau_{0}<\infty\right)=1$, as claimed.

\section{Unique invariant probability measure for $\Theta$ and proof of Theorem 2.2}

We show that the time-changed angular part $\Theta$ of $X$ is the unique solution to a martingale problem. This is a non-trivial consequence of our uniqueness assumption on $X$. The difficulty is due to the lack of a skew product representation: the radial and angular parts can interact in a complicated manner. Once uniqueness of $\Theta$ is established, we will prove Theorem 2.2 with the aid of the following theorem. It is a compilation of results taken from Chapter I, Sections 1.2 and 4.1, in Skorokhod (1989).

Theorem 3.1. Suppose $K$ is a compact metric space and $\tilde{X}$ is a continuous homogeneous 
Markov process with Feller transition probability function $P(t, x, A)$; that is, for each $f \in C(K)$,

$$
T_{t} f(x)=\int_{K} f(y) P(t, x, \mathrm{~d} y) \in C(K) .
$$

Suppose for some probability measure $\pi(\mathrm{d} y)$ with support dense in $K$, for some $q>0$,

$$
\int_{0}^{\infty} \mathrm{e}^{-q t} P(t, x, \cdot) \mathrm{d} t
$$

is absolutely continuous with respect to $\pi$ for each $x \in K$. Then $\tilde{X}$ has a unique invariant probability measure $v$ absolutely continuous with respect to $\pi$ and

$$
\lim _{t \rightarrow \infty} \frac{1}{t} \int_{0}^{f} f\left(\tilde{X}_{s}\right) \mathrm{d} s=\int_{K} f(x) v(\mathrm{~d} x) \text { a.s. }
$$

for all bounded measurable $f$ on $K$.

Henceforth we will use the following terminology. Given a manifold $M$ and a differential operator $\mathscr{D}$ on $C_{0}^{\infty}(M)$, we say a probability measure $\mathscr{P}$ on $C([0, \infty), M)$ (equipped with the Borel $\sigma$-algebra and filtration generated by the coordinate process) solves the $\tau$-stopped martingale problem for $\mathscr{D}$, starting from $m \in M$, if $\tau$ is a stopping time, $\mathscr{P}\left(\omega_{0}=m\right)=1$ and, for each $f \in C_{0}^{\infty}(M)$,

$$
f(\omega(t \wedge \tau))-\int_{0}^{t \wedge \tau}(\mathscr{D} f)\left(\omega_{s}\right) \mathrm{d} s
$$

is a $\mathscr{P}$-martingale. If $\tau \equiv \infty$, we say $\mathscr{P}$ solves the $\mathscr{D}$-martingale problem.

In the polar coordinates $(\theta, r)$, where $r=|x|$ and $\theta(x)$ is the point on $S^{d-1}$ corresponding to $x /|x|$,

$$
L=\left[\frac{1}{2} \frac{\partial^{2}}{\partial r^{2}}+\frac{1}{2} \frac{1}{r}\left[A(\theta)^{-1}\{\operatorname{tr} a(\theta)+G(\theta)\}-1\right] \frac{\partial}{\partial r}+\frac{1}{r} \frac{\partial}{\partial r} \bar{L}_{1}+\frac{1}{r^{2}} \bar{L}_{2}\right],
$$

where $\bar{L}_{1}$ and $\bar{L}_{2}$ are from (1.6), $A$ from (1.7) and $G$ from the line after (2.7). The following theorem gives a characterization of $\Theta$.

Theorem 3.2. For each $x \neq 0$, the law of $\Theta$ under $\bar{P}_{x}^{0}$ on $C\left([0, \infty), S^{d-1}\right)$ is the unique solution to the $\bar{L}_{2}$-martingale problem starting from $x /|x|$. In particular, $\Theta$ is a Markov process.

Proof. We only consider $d \geqslant 3$. The two-dimensional case is much simpler and we leave the details to the reader. The Markov property is a consequence of uniqueness (see Theorem 4.2 of Ethier and Kurtz 1986, p. 184). To prove existence of a unique solution to the $\bar{L}_{2-}$ martingale problem starting from $x /|x|$, we use the coordinates on $S^{d-1}$ induced by stereographic projection. The advantage is that only two charts are needed to cover $S^{d-1}$ : the projections from two distinct points on $S^{d-1}$. The projection $\Psi$ from the north pole is given by 


$$
\Psi(x)=\left(\frac{x_{1}}{1-x_{d}}, \ldots, \frac{x_{d-1}}{1-x_{d}}\right), \quad x=\left(x_{1}, \ldots, x_{d}\right) \in \mathbb{R}^{d},|x|=1, x_{d} \neq 1 .
$$

The inverse mapping is

$$
\Psi^{-1}\left(y_{1}, \ldots, y_{d-1}\right)=\left(\frac{2 y_{1}}{|y|^{2}+1}, \ldots, \frac{2 y_{d-1}}{|y|^{2}+1}, \frac{|y|^{2}-1}{|y|^{2}+1}\right), \quad y \in \mathbb{R}^{d-1} .
$$

Our polar coordinates take on the (local) form $(y, r)$, where

$$
r=|x|, \quad y=\Psi(x /|x|), \quad x \neq 0 .
$$

Note that the inverse mapping is

$$
x=r \Psi^{-1}(y), \quad r>0 .
$$

Let $\tilde{L}$ be $\bar{L}$ expressed in the coordinates $(y, r)$ :

$$
\tilde{L}=\frac{1}{2} \frac{\partial^{2}}{\partial r^{2}}+\frac{1}{r} \tilde{b}_{d}(y) \frac{\partial}{\partial r}+\frac{1}{r} \sum_{l=1}^{d-1} \tilde{a}_{l d}(y) \frac{\partial^{2}}{\partial y_{l} \partial r}+\frac{1}{r^{2}}\left[\frac{1}{2} \sum_{k, l=1}^{d-1} \tilde{a}_{k l}(y) \frac{\partial^{2}}{\partial y_{k} \partial y_{l}}+\sum_{l=1}^{d-1} \tilde{b}_{l}(y) \frac{\partial}{\partial y_{l}}\right]
$$

where, for $k, l=1, \ldots, d-1$,

$$
\begin{aligned}
& \tilde{b}_{l}(y)=\left\{\frac{|x|^{2}}{2} \sum_{i, j=1}^{d} a_{i j}(x) \frac{\partial^{2} y_{l}}{\partial x_{i} \partial x_{j}}+\left(b(x), \nabla y_{l}\right)|x|\right\} /\left[(a(x) x, x) /|x|^{2}\right], \\
& \tilde{b}_{d}(y)=\left\{\frac{1}{2}\left[\operatorname{tr} a(x)-\frac{(a(x) x, x)}{|x|^{2}}\right]+(x, b(x)) /|x|\right\} /\left[(a(x) x, x) /|x|^{2}\right], \\
& \tilde{a}_{l d}(y)=\tilde{a}_{d l}(y)=\left(a(x) x, \nabla y_{l}\right) /\left[(a(x) x, x) /|x|^{2}\right], \\
& \tilde{a}_{k l}(y)=\tilde{a}_{l k}(y)=|x|^{2}\left(a(x) \nabla y_{k}, \nabla y_{l}\right) /\left[(a(x) x, x) /|x|^{2}\right]
\end{aligned}
$$

are locally bounded and independent of $r$, since $a(x), b(x), r \nabla y_{l}$ and $r^{2}\left(\partial^{2} y_{l} / \partial x_{i} \partial x_{j}\right)$ depend only on $x /|x|$. Hence in $(y, r)$ coordinates, the operator $\bar{L}_{2}$ in (3.1) takes the form

$$
\tilde{L}_{2}=\frac{1}{2} \sum_{k, l=1}^{d-1} \tilde{a}_{k l}(y) \frac{\partial^{2}}{\partial y_{k} \partial y_{l}}+\sum_{l=1}^{d-1} \tilde{b}_{l}(y) \frac{\partial}{\partial y_{l}} .
$$

Consider any $x \neq 0$. It is no loss to assume $x /|x|$ is not the north pole (otherwise, use stereographic projection from the south pole). To prove existence and uniqueness of a solution to the $\bar{L}_{2}$-martingale problem starting from $x /|x|$, we first prove local existence and uniqueness. Then we show explosion does not occur, and the proof will be complete. We now verify $\tilde{a}$ is uniformly positive definite. For $r=|x| \neq 0$ and $y=\Psi(\mathrm{x} /|\mathrm{x}|)$, 


$$
\begin{aligned}
\sum_{k, l=1}^{d-1}\left(a(x) \nabla y_{k}, \nabla y_{l}\right) y_{k} y_{l} & =\sum_{k, l=1}^{d-1}\left(a(x) \nabla \frac{y_{k}^{2}}{2}, \nabla \frac{y_{l}^{2}}{2}\right) \\
& =\sum_{k, l=1}^{d-1} \sum_{i, j=1}^{d} a_{i j}(x)\left[\frac{\partial}{\partial x_{i}}\left(\frac{y_{k}^{2}}{2}\right)\right]\left[\frac{\partial}{\partial x_{j}}\left(\frac{y_{k}^{2}}{2}\right)\right] \\
& =\sum_{i, j=1}^{d} a_{i j}(x)\left[\frac{\partial}{\partial x_{i}} \frac{|y|^{2}}{2}\right]\left[\frac{\partial}{\partial x_{j}} \frac{|y|^{2}}{2}\right], \quad\left(|y|^{2}=\sum_{l=1}^{d-1} y_{l}^{2}\right) \\
& \geqslant \lambda\left|\nabla \frac{|y|^{2}}{2}\right|^{2} \text { (by uniform ellipticity of } a \text { ). }
\end{aligned}
$$

But

$$
|y|^{2}=\sum_{l=1}^{d-1} y_{l}^{2}=\sum_{l=1}^{d-1} \frac{x_{l}^{2}}{\left(x_{d}-r\right)^{2}}=\frac{r^{2}-x_{d}^{2}}{\left(r-x_{d}\right)^{2}}=\frac{r+x_{d}}{r-x_{d}}
$$

and so

$$
\begin{aligned}
\left.\left.|\nabla| y\right|^{2}\right|^{2} & =\sum_{i=1}^{d}\left(\frac{\partial}{\partial x_{i}} \frac{r+x_{d}}{r-x_{d}}\right)^{2} \\
& =\sum_{i=1}^{d} \frac{4}{r^{2}\left(r-x_{d}\right)^{4}}\left[r^{2} \delta_{i d}-x_{i} x_{d}\right]^{2} \\
& =\frac{4}{r^{2}\left(r-x_{d}\right)^{4}}\left[r^{4}-2 x_{d}^{2} r^{2}+r^{2} x_{d}^{2}\right] \\
& =\frac{4}{\left(r-x_{d}\right)^{4}}\left[r^{2}-x_{d}^{2}\right]^{2} \\
& =\frac{4\left(r+x_{d}\right)}{\left(r-x_{d}\right)^{3}} \\
& =\frac{4|y|^{2}}{\left(r-x_{d}\right)^{2}} .
\end{aligned}
$$

Using (3.5) and (3.3),

$$
r-x_{d}=r-r \frac{|y|^{2}-1}{|y|^{2}+1}=\frac{2 r}{|y|^{2}+1}
$$

and so

$$
\left.\left.|\nabla| y\right|^{2}\right|^{2}=\frac{|y|^{2}}{r^{2}}\left[|y|^{2}+1\right]^{2} \geqslant \frac{|y|^{2}}{r^{2}} .
$$


Thus

$$
r^{2} \sum_{k, l=1}^{d-1}\left(a(x) \nabla y_{k}, \nabla y_{l}\right) y_{k} y_{l} \geqslant \frac{\lambda}{4} \cdot|y|^{2} \text {. }
$$

In particular, for some constant $\tilde{\lambda}>0$

$$
\sum_{k, l=1}^{d-1} \tilde{a}_{k l}(y) y_{k} y_{l} \geqslant \tilde{\lambda}|y|^{2}, \quad y \in \mathbb{R}^{d-1},
$$

and $\tilde{a}$ is uniformly positive definite. Hence a solution to the $\tilde{L}_{2}$-martingale problem exists locally. More precisely, given any bounded open set $G \subseteq \mathbb{R}^{d-1}$, if $\tilde{\tau}_{G}$ is the first exit time from $G$, then the $\tilde{\tau}_{G}$-stopped martingale problem for $\tilde{L}_{2}$ has a solution.

Local uniqueness for the $\tilde{L}_{2}$-martingale problem. Let $G \subseteq \mathbb{R}^{d-1}$ be a bounded open set. We show that the $\tilde{\tau}_{G}$-stopped martingale problem for $\tilde{L}_{2}$ has a unique solution. The values of $\tilde{a}$ and $\tilde{b}$ off $\bar{G}$ are immaterial, so it is no loss to assume $\tilde{b}=0$ and $\tilde{a}=I$ off $\bar{G}$.

Suppose $\tilde{P}_{y}$ is a solution to the $\tilde{\tau}_{G}$-stopped martingale problem for $\tilde{L}_{2}$ starting from $y=\Psi(x /|x|)$. We will construct processes $R_{t}$ and $Y_{t}$ such that the law of $Y_{t}$ is $\tilde{P}_{y}$ and a time change of $R_{t} \Psi^{-1}\left(Y_{t}\right)$ furnishes a process $\bar{X}_{t}$ whose law solves the $\bar{L}$-martingale problem stopped upon exiting the cone $\mathscr{C}=\left\{r \Psi^{-1}(y): r>0, y \in G\right\}$. Writing $\tau_{\mathscr{C}}$ for this time, we see $\tau_{\mathscr{C}} \leqslant \tau_{0}$. By our basic assumption, the $\tau_{0}$-stopped martingale problem for $\bar{L}$ has a unique solution, so the law of $\bar{X}_{t}$ must be the unique solution to the $\bar{\tau}_{\mathscr{C}}$-stopped martingale problem for $\bar{L}$. Then $\tilde{P}_{y}$ is uniquely determined, provided the time change is reasonable. Now for the details.

For some $(d-1)$-dimensional Brownian motion $\tilde{W}$ on $\left(\Omega_{d-1}, \mathscr{F}, \tilde{P}_{y}\right)$, if $Y$ is the coordinate process on $\Omega_{d-1}$, then for $t \leqslant \tilde{\tau}_{G}$,

$$
Y_{t}=y+\int_{0}^{t} \tilde{a}^{1 / 2}\left(Y_{s}\right) \mathrm{d} \tilde{W}_{s}+\int_{0}^{t}\left(\tilde{b}_{1}, \ldots, \tilde{b}_{d-1}\right)^{*}\left(Y_{s}\right) \mathrm{d} s,
$$

where $\tilde{a}^{1 / 2}$ is the positive definite symmetric square root of $\tilde{a}$. Since $\tilde{a}=I$ and $\tilde{b}=0$ off $\bar{G}$, this equation makes sense for all $t \geqslant 0$.

In the coordinates $(y, r)$, the matrix of coefficients for the second-order part of $r^{2} \tilde{L}$ (from (3.6)) can be written as

$$
\frac{1}{2}\left(\begin{array}{cc}
\tilde{a}(y) & r F^{*}(y) \\
r F(y) & r^{2}
\end{array}\right)
$$

where $F$ is a $1 \times(d-1)$ matrix and ${ }^{*}$ denotes transpose. As in Lemma 4.2 of Bass and Pardoux (1987), there is a $d \times d$ matrix $\tilde{\sigma}$ of the form

$$
\tilde{\sigma}(y, r)=\left(\begin{array}{cc}
\tilde{a}^{1 / 2}(y) & 0 \\
r E(y) & r C(y)
\end{array}\right)
$$

such that 


$$
\tilde{\sigma} \tilde{\sigma}^{*}=\left(\begin{array}{cc}
H & r F^{*} \\
r F & r^{2}
\end{array}\right)
$$

in fact, $E=F \tilde{a}^{-1 / 2}, C=\sqrt{1-E E^{*}}>0$.

Let $W_{d}(t)$ be a one-dimensional Brownian motion independent of $\tilde{W}$. Then since $E E^{*}+C^{2}=1$, the quadratic variation of

$$
\mathscr{B}_{t}:=\int_{0}^{t} E\left(Y_{s}\right) \mathrm{d} \tilde{W}_{s}+\int_{0}^{t} C\left(Y_{s}\right) \mathrm{d} W_{d}(s)
$$

is

$$
\langle\mathscr{B}, \mathscr{B}\rangle_{t}=t .
$$

Thus $\mathscr{B}_{t}$ is one-dimensional Brownian motion. Setting

$$
R_{t}=r \exp \left\{\mathscr{B}_{t}+\int_{0}^{t} b_{d}\left(Y_{s}\right) \mathrm{d} s-t / 2\right\}, \quad r=|x|,
$$

by Itô's formula $R_{t}$ satisfies

$$
R_{t}=r+\int_{0}^{t} R_{s} \mathrm{~d} \mathscr{B}_{s}+\int_{0}^{t} R_{s} b_{d}\left(Y_{s}\right) \mathrm{d} s .
$$

Hence for $f_{1} \in C_{0}^{\infty}\left(\mathbb{R}^{d-1}\right)$ and $f_{2} \in C_{0}^{\infty}((0, \infty))$, setting $f(y, r)=f_{1}(y) f_{2}(r)$, by Itô's formula

$$
f\left(Y_{t}, R_{t}\right)-\int_{0}^{t}\left[\left(r^{2} \tilde{L}\right) f\right]\left(Y_{s}, R_{s}\right) \mathrm{d} s
$$

is a martingale. Define

$$
\phi_{t}=\int_{0}^{t} R_{s}^{2} \mathrm{~d} s
$$

and observe that $\phi:[0, \infty) \rightarrow[0, \phi(\infty))$ is strictly increasing and continuous. Therefore $\phi$ has a continuous strictly increasing inverse $\phi_{t}^{-1}:[0, \phi(\infty)) \rightarrow[0, \infty)$. Set

$$
\begin{gathered}
\left(\bar{Y}_{t}, \bar{R}_{t}\right)=\left(Y\left(\phi_{t}^{-1}\right), R\left(\phi_{t}^{-1}\right)\right), \quad t<\phi(\infty), \\
\bar{X}_{t}=\bar{R}_{t} \Psi^{-1}\left(\bar{Y}_{t}\right), \quad t<\phi(\infty) .
\end{gathered}
$$

Since $G \subseteq \mathbb{R}^{d-1}$ is bounded, it is not hard to see $\tilde{\tau}_{G}<\infty$ almost surely and hence

$$
\phi\left(\tilde{\tau}_{G}\right)<\phi(\infty) \text { a.s. }
$$

With $f$ as above, for $\delta>0$, 


$$
\begin{aligned}
& f\left(\bar{Y}\left(t \wedge \phi\left(\tilde{\tau}_{G}\right)\right), \bar{R}\left(t \wedge \phi\left(\tilde{\tau}_{G}\right)\right)\right)-\int_{0}^{t \wedge \phi\left(\tilde{\tau}_{G}\right)}(\tilde{L} f)\left(\bar{Y}_{s}, \bar{R}_{s}\right) \mathrm{d} s \\
& =f(Y, R) \circ \phi^{-1}\left(t \wedge \phi\left(\tilde{\tau}_{G}\right)\right)-\int_{0}^{t \wedge \phi\left(\tilde{\tau}_{G}\right)}(\tilde{L} f)\left(Y_{\phi_{s}^{-1}}, R_{\phi_{s}^{-1}}\right) \mathrm{d} s \\
& =f(Y, R) \circ \phi^{-1}\left(t \wedge \phi\left(\tilde{\tau}_{G}\right)\right)-\int_{0}^{\phi^{-1}\left(t \wedge \phi\left(\tilde{\tau}_{G}\right)\right)}\left[\left(r^{2} \tilde{L}\right) f\right]\left(Y_{u}, R_{u}\right) \mathrm{d} u
\end{aligned}
$$

is a martingale, by (3.9), optional stopping and dominated convergence. Hence $\left(\bar{Y}\left(t \wedge \phi\left(\tilde{\tau}_{G}\right)\right)\right.$, $\left.\bar{R}\left(t \wedge \phi\left(\tilde{\tau}_{G}\right)\right)\right)$ solves the $\phi\left(\tilde{\tau}_{G}\right)$-stopped martingale problem for $\tilde{L}$. Since $\tilde{L}$ is $\bar{L}$ expressed in the coordinates $(y, r)$, we see that for each $\delta>0$, the law of $\bar{X}_{t}$ solves the $\phi\left(\tilde{\tau}_{G}\right)$-stopped martingale problem for $\bar{L}$. By (3.8) $R(\cdot)$ does not hit 0 in finite time and consequently $\bar{R}(\cdot)=|\bar{X}(\cdot)|$ does not hit 0 before $\phi(\infty)$. Then by (3.10) and our basic assumption of uniqueness for the $\tau_{0}$-stopped martingale problem for $\bar{L}$, the law of $\bar{X}\left(\cdot \wedge \phi\left(\tilde{\tau}_{G}\right)\right)$ is uniquely determined. Also, for $t<\phi(\infty)$, the inverse of $\phi$ is

$$
\phi_{t}^{-1}=\int_{0}^{t} \bar{R}_{u}^{-2} \mathrm{~d} u,
$$

and it follows that the law of $\bar{X}\left(\phi(\cdot) \wedge \phi\left(\tilde{\tau}_{G}\right)\right)=\bar{X}\left(\phi\left(\cdot \wedge \tilde{\tau}_{G}\right)\right)$ is uniquely determined. Hence the law of

$$
Y\left(\cdot \wedge \tilde{\tau}_{G}\right)=\bar{Y}\left(\phi\left(\cdot \wedge \tilde{\tau}_{G}\right)\right)
$$

is uniquely determined, as claimed.

Non-explosion. We have shown a solution to the $\tilde{L}_{2}$-martingale problem exists uniquely up to an explosion time. Note that explosion in finite time for this solution corresponds to a solution to the $\bar{L}_{2}$-martingale problem hitting the north pole in finite time. Hence the $\bar{L}_{2}$ martingale problem has a unique solution up to the first hitting time $\gamma_{1}$ of the north pole. By repeating the preceding proof using stereographic projection from the south pole, we can extend the solution uniquely to the first time $\gamma_{2}$ after $\gamma_{1}$ the south pole is hit. Repeating this, we obtain a unique solution up to the explosion time $\gamma_{\infty}=\sup _{n} \gamma_{n}$, where

$$
\begin{aligned}
\gamma_{2 n} & =\inf \left\{\mathrm{t}>\gamma_{2 n-1}: \text { hit south pole }\right\}, \\
\gamma_{2 n+1} & =\inf \left\{\mathrm{t}>\gamma_{2 n}: \text { hit north pole }\right\} .
\end{aligned}
$$

Stated another way, the $\gamma_{\infty}$-stopped martingale problem for $\bar{L}_{2}$ has a unique solution. Write $Q_{\theta}$ for this solution.

To see that explosion does not occur (that is, $\gamma_{\infty=} \infty$ ) proceed as follows. By uniqueness, the family $\left\{Q_{\theta}: \theta \in S^{d-1}\right\}$ possesses the strong Markov property. Denote

$$
\begin{aligned}
& \gamma_{\mathrm{NP}}=\inf \left\{\mathrm{t} \geqslant 0: \omega_{t}=\text { north pole }\right\} \\
& \gamma_{\mathrm{SP}}=\inf \left\{\mathrm{t} \geqslant 0: \omega_{t}=\text { south pole }\right\},
\end{aligned}
$$

where $\omega_{t}$ is the coordinate process. By the support theorem, for $T>0$, 


$$
Q_{\mathrm{NP}}\left(\gamma_{\mathrm{SP}}<T\right)<1
$$

Hence, for $\theta \in S^{d-1}$ and $T>0$, by the strong Markov property

$$
\begin{aligned}
Q_{\theta}\left(\gamma_{\infty}<T\right) & \leqslant Q_{\theta}\left(\gamma_{2 n}<T\right) \\
& =Q_{\theta}\left(\gamma_{2 n-1}<T, \gamma_{2 n}<T\right) \\
& \leqslant Q_{\theta}\left(\gamma_{2 n-1}<T\right) Q_{\mathrm{NP}}\left(\gamma_{\mathrm{SP}}<T\right) \\
& \leqslant Q_{\theta}\left(\gamma_{2 n-2}<T\right) Q_{\mathrm{NP}}\left(\gamma_{\mathrm{SP}}<T\right) \\
& \vdots \\
& \leqslant Q_{\theta}\left(\gamma_{2}<T\right) Q_{\mathrm{NP}}\left(\gamma_{\mathrm{SP}}<T\right)^{n-1} \\
& \leqslant Q_{\mathrm{NP}}\left(\gamma_{\mathrm{SP}}<T\right)^{n-1}
\end{aligned}
$$

Letting $n \rightarrow \infty$ yields

$$
Q_{\theta}\left(\gamma_{\infty}<T\right)=0
$$

Since $T>0$ was arbitrary, we have

$$
Q_{\theta}\left(\gamma_{\infty}<\infty\right)=0,
$$

as desired. This completes the proof of Theorem 3.2.

We will need the next lemma to prove Feller continuity. Its proof uses the notation from the proof of the last theorem.

Lemma 3.3. Let $U$ be an open subset of $S^{d-1}$ whose closure does not contain the north pole and set $\tau_{U}=\inf \left\{t \geqslant 0: \omega_{t} \notin U\right\}$. If $Q$ solves the $\bar{L}_{2}$-martingale problem starting from $\theta \in U$ then

$$
\mathrm{E}^{Q}\left[\int_{0}^{\tau_{U}} I\left(\omega_{t} \in D\right) \mathrm{d} t\right]=0,
$$

where $D$ is the set of discontinuities of $a$ and $b$ regarded as functions on $S^{d-1}$.

Proof. Let $y=\Psi(\theta)$ and $G=\Psi(U)$. If $\tilde{Q}$ is the solution to the $\tilde{\tau}_{G}$-stopped martingale problem for $\tilde{L}_{2}$, then (3.12) is equivalent to

$$
\mathrm{E}^{\tilde{Q}}\left[\int_{0}^{\tilde{\tau}_{G}} I\left(\omega_{t} \in \Psi(D)\right) \mathrm{d} t\right]=0 .
$$

Since $\bar{U}$ does not contain the north pole, $G$ is a bounded open set in $\mathbb{R}^{d-1}$, so by a theorem of Krylov (1971), there exists $N>0$ independent of $y$ such that for any Borel function $\tilde{g}$, 


$$
\mathrm{E}^{\tilde{Q}}\left[\int_{0}^{\tilde{\tau}_{G}} \tilde{g}\left(\omega_{t}\right) \mathrm{d} t\right] \leqslant N\left[\int_{G}|\tilde{g}(x)|^{d-1} \mathrm{~d} x\right]^{1 /(d-1)} .
$$

In particular, by (1.5) the $(d-1)$-dimensional Lebesgue measure of $\Psi(D)$ is zero, so (3.13) is immediate.

The next step is to verify the Feller property.

Lemma 3.4. The process $\Theta$ is Feller; that is, for each $f \in C\left(S^{d-1}\right)$ and $t>0$,

$$
x /|x| \in S^{d-1} \rightarrow \mathrm{E}^{\bar{P}_{x}^{0}}\left[f\left(\Theta_{t}\right)\right]
$$

is continuous.

Proof. Let $x_{n}, x \in \mathbb{R}^{d} \backslash\{0\} \quad$ with $\quad x_{n} /\left|x_{n}\right| \rightarrow x /|x|$. It is no loss to assume $x$, $\left.x_{n} \in \mathbb{R}^{d} \backslash\{0, \ldots, 0, a): a \geqslant 0\right\}$. Let $Q$ and $Q_{n}$ denote the laws on $C\left([0, \infty), S^{d-1}\right)$ of $\Theta$ under $\bar{P}_{x}^{0}$ and $\bar{P}_{x_{n}}^{0}$, respectively. Thus we need to prove

$$
Q_{n} \rightarrow \mathscr{L} Q \text { as } n \rightarrow \infty \text {. }
$$

Since $S^{d-1}$ is compact, we can argue much like the proof of Theorem 1.4.6 in Stroock and Varadhan (1979) to show $\left\{Q_{n}: n \geqslant 1\right\}$ is precompact. (Note that we replace 'translates of $f$ ' in their hypothesis 1.4 .3 by 'rotations of $f$ '.) Let $Q$ be any limit point and suppose $Q_{n_{k}} \rightarrow \mathscr{L} Q$. Once we show $\mathcal{Q}=Q$, it follows that any subsequence of $\left\{Q_{n}\right\}$ contains a subsubsequence converging to $Q$. Then $Q_{n} \rightarrow \mathscr{L} Q$, as desired.

From the proof of the Theorem 3.2, it is enough to show $Q$ solves the $\tau_{U}$-stopped martingale problem for $\bar{L}_{2}$, where $U$ is an open subset of $S^{d-1}$ whose closure does not contain the north pole. Let $x(\omega, t)=\omega_{t}$ be the coordinate mapping on $C\left([0, \infty), S^{d-1}\right)$ and set $\mathscr{C}_{t}=\sigma(\mathrm{x}(\mathrm{s}): s \leqslant t)$. Consider any $f \in C^{\infty\left(S^{d-1}\right)}$ that is constant in a neighbourhood of the north pole. Recalling that $D$ is the set of discontinuities of $a$ and $b$ regarded as functions on $S^{d-1}$ and that the surface measure of $D$ is 0 (see (1.5)), we see that

$$
\omega \rightarrow \int_{0}^{t}\left(\bar{L}_{2} f\right)\left(\omega_{s}\right) \mathrm{d} s
$$

is continuous on the set

$$
\mathscr{D}^{c}=\left\{\omega: \int_{0}^{t} I\left(\omega_{s} \in D\right) \mathrm{d} s=0\right\} .
$$

(Here the superscript $c$ stands for complement.) If

$$
Q(\mathscr{D})=0
$$

then by the continuous mapping theorem, for each bounded continuous $\mathscr{L}_{s}$-measurable function $\Phi$ on $C\left([0, \infty), S^{d-1}\right)$ and $t \geqslant s$, 


$$
\begin{aligned}
\mathrm{E}^{\mathcal{Q}}\left[\left\{f\left(\omega_{t}\right)-\int_{0}^{t}\left(\bar{L}_{2} f\right)\left(\omega_{u}\right) \mathrm{d} u\right\} \Phi\right] & =\lim _{k \rightarrow \infty} \mathrm{E}^{Q_{n_{k}}}\left[\left\{f\left(\omega_{t}\right)-\int_{0}^{t}\left(\bar{L}_{2} f\right)\left(\omega_{u}\right) \mathrm{d} u\right\} \Phi\right] \\
& =\lim _{k \rightarrow \infty} \mathrm{E}^{Q_{n_{k}}}\left[\left\{f\left(\omega_{s}\right)-\int_{0}^{s}\left(\bar{L}_{2} f\right)\left(\omega_{u}\right) \mathrm{d} u\right\} \Phi\right] \\
& =\mathrm{E}^{\mathcal{Q}}\left[\left\{f\left(\omega_{s}\right)-\int_{0}^{s}\left(\bar{L}_{2} f\right)\left(\omega_{u}\right) \mathrm{d} u\right\} \Phi\right] .
\end{aligned}
$$

Thus $f\left(\omega_{t}\right)-\int_{0}^{t}\left(\bar{L}_{2} f\right)\left(\omega_{u}\right) \mathrm{d} u$ is a $Q$-martingale and so $\mathcal{Q}$ solves the $\tau_{U}$-stopped $\bar{L}_{2}$ martingale problem, as desired.

To verify (3.14), note that it is enough to show

$$
\mathrm{E}^{\mathcal{Q}}\left[\int_{0}^{\tau_{U}} I\left(\omega_{t} \in D\right) \mathrm{d} t\right]=0,
$$

where $U$ is any open subset of $S^{d-1}$ whose closure does not contain the north pole. It is a simple matter to show that the function

$$
\omega \rightarrow \int_{0}^{\tau_{U}} I\left(\omega_{t} \in D\right) \mathrm{d} t
$$

is lower semicontinuous and hence

$$
\begin{aligned}
\mathrm{E}^{\mathcal{Q}}\left[\int_{0}^{\tau_{U}} I\left(\omega_{t} \in D\right) \mathrm{d} t\right] & \leqslant \varliminf_{k \rightarrow \infty} \mathrm{E}^{Q_{n_{k}}}\left[\int_{0}^{\tau_{U}} I\left(\omega_{t} \in D\right) \mathrm{d} t\right] \\
& =0
\end{aligned}
$$

by Lemma 3.3 .

Now we verify the absolute continuity hypothesis in Theorem 3.1 .

Lemma 3.5. For $x \neq 0$ and $q>0$,

$$
\int_{0}^{\infty} \mathrm{e}^{-q t} \bar{P}_{x}^{0}\left(\Theta_{t} \in \cdot\right) \mathrm{d} t
$$

is absolutely continuous with respect to the normalized surface measure $\mu$ on $S^{d-1}$.

Proof. It is no loss to assume $x /|x|$ is not the north pole. Then for any Borel set $A \subseteq S^{d-1}$, in the notation of the proof of Theorem 3.2,

$$
\bar{P}_{x}^{0}\left(\Theta_{t} \in A\right)=\tilde{P}_{y}\left(Y_{t} \in \Psi(A)\right), \quad y=\Psi(x /|x|) .
$$

If $\mu(A)=0$ then the $(d-1)$-dimensional Lebesgue measure of $\Psi(A)$ is 0 . Hence by Krylov's theorem cited above,

$$
\int_{0}^{\infty} I\left(Y_{s} \in \Psi(A)\right) \mathrm{d} s=0 \text { a.s. } \tilde{P}_{y} .
$$


Then

$$
\begin{aligned}
0 & =\int_{0}^{\infty} \mathrm{e}^{-q t} \tilde{P}_{y}\left(Y_{t} \in \Psi(A)\right) \mathrm{d} t \\
& =\int_{0}^{\infty} \mathrm{e}^{-q t} \bar{P}_{x}^{0}\left(\Theta_{t} \in A\right) \mathrm{d} t
\end{aligned}
$$

as desired.

By Theorem 3.2 and Lemmas 3.4-3.5, the hypotheses of Theorem 3.1 hold for $K=S^{d-1}$ and $\tilde{X}=\Theta$. Then Theorem 3.1 immediately yields Theorem 2.2.

\section{The invariant measure for $d=2$}

Let $d=2$ and suppose $b_{i} \equiv 0, i=1,2$. Assume $a$ is piecewise smooth on $S^{1}$ : that is, for some $0=\theta_{0}<\theta_{1}<\cdots<\theta_{n}=2 \pi,\left.a\right|_{\left(\theta_{i-1}, \theta_{i}\right)}$ has a smooth extension to $\left[\theta_{i-1}, \theta_{i}\right]$, $i=1, \ldots, n$. Writing $L$ in polar coordinates $x=r \mathrm{e}^{\mathrm{i} \theta}$,

$$
L=\frac{1}{2}\left\{\mathrm{e}_{1} \frac{\partial^{2}}{\partial r^{2}}+\frac{e_{3}}{r} \frac{\partial^{2}}{\partial \theta \partial r}+\frac{e_{2}}{r} \frac{\partial}{\partial r}+\frac{1}{r^{2}}\left[\mathrm{e}_{2} \frac{\partial^{2}}{\partial \theta^{2}}-\mathrm{e}_{3} \frac{\partial}{\partial \theta}\right]\right\},
$$

where

$$
\begin{aligned}
& e_{1}=\frac{\langle a(x) x, x\rangle}{|x|^{2}}=a_{11} \cos ^{2} \theta+2 a_{12} \cos \theta \sin \theta+a_{22} \sin ^{2} \theta \\
& e_{2}=a_{11} \sin ^{2} \theta-2 a_{12} \cos \theta \sin \theta+a_{22} \cos ^{2} \theta \\
& e_{3}=-2 a_{11} \cos \theta \sin \theta+2 a_{22} \cos \theta \sin \theta+2\left(\cos ^{2} \theta-\sin ^{2} \theta\right) a_{12} .
\end{aligned}
$$

Then

$$
\bar{L}_{2}=\frac{1}{2}\left[\frac{e_{2}}{e_{1}} \frac{\partial^{2}}{\partial \theta^{2}}-\frac{e_{3}}{e_{1}} \frac{\partial}{\partial \theta}\right] .
$$

It is easy to show that the invariant probability measure $v$ from Theorem 2.2 is absolutely continuous with respect to arc length $\mathrm{d} \theta$ on $S^{1}$. Thus for some $p(\theta)$,

$$
v(\mathrm{~d} \theta)=p(\theta) \mathrm{d} \theta \text {. }
$$

The main result of this section is the explicit identification of $p(\theta)$. Define

$$
h(u)=\exp \left\{\int_{0}^{u}-e_{3}(\theta) / \mathrm{e}_{2}(\theta) \mathrm{d} \theta\right\} .
$$

Theorem 4.1. The invariant density $p(\theta)$ is given by 


$$
p(\theta)= \begin{cases}B e_{1}(\theta) h(\theta) / \mathrm{e}_{2}(\theta), & \text { if } h(2 \pi)=1 \\ B e_{1}(\theta) h(\theta) / \mathrm{e}_{2}(\theta)\left[\int_{0}^{\theta} h(u)^{-1} \mathrm{~d} u+\frac{h(2 \pi)}{1-h(2 \pi)} \int_{0}^{2 \pi} h(u)^{-1} \mathrm{~d} u\right], & \text { if } h(2 \pi) \neq 1\end{cases}
$$

where $\theta \notin\left\{\theta_{1}, \ldots, \theta_{n}\right\}$ and $B$ is chosen so $\int_{0}^{2 \pi} p(\theta) \mathrm{d} \theta=1$.

Proof. Let $E_{\theta}$ denote expectation for the process $\Theta_{t}$ starting at $\theta$ corresponding to $\bar{L}_{2}$ on $S^{1}$. Then for smooth enough functions $f$ defined on $S^{1}$,

$$
\mathrm{E}_{\theta} f\left(\Theta_{t}\right)-f(\theta)=\mathrm{E}_{\theta} \int_{0}^{t}\left(\bar{L}_{2} f\right)\left(\Theta_{s}\right) \mathrm{d} s .
$$

Integrating with respect to $v=p \mathrm{~d} \theta$,

$$
0=t \int_{0}^{2 \pi}\left(\bar{L}_{2} f\right)(\theta) p(\theta) \mathrm{d} \theta
$$

Then writing $\tilde{p}=p / e_{1}$,

$$
\int_{0}^{2 \pi}\left(e_{2} \frac{\partial^{2} f}{\partial \theta^{2}}-e_{3} \frac{\partial f}{\partial \theta}\right) \tilde{p} \mathrm{~d} \theta=0 .
$$

Since the coefficients of $e_{1} \bar{L}_{2}$ are in $C^{\infty}\left(\theta_{i-1}, \theta_{i}\right), \tilde{p} \in C^{\infty}\left(\theta_{i-1}, \theta_{i}\right)$. Integrating by parts twice yields, for $f \in C^{\infty}$,

$$
\begin{aligned}
0= & \sum_{i=1}^{n}\left[\left\{\left(\tilde{p} \mathrm{e}_{2} f^{\prime}\right)\left(\theta_{i}^{-}\right)-\left(\tilde{p} \mathrm{e}_{2} f^{\prime}\right)\left(\theta_{i-1}^{+}\right)\right\}-\left\{\left[\tilde{p} \mathrm{e}_{3}+\left(\tilde{p} \mathrm{e}_{2}\right)^{\prime}\right] f\left(\theta_{i}^{-}\right)-\left[\tilde{p} \mathrm{e}_{3}+\left(\tilde{p} \mathrm{e}_{2}\right)^{\prime}\right] f\left(\theta_{i-1}^{+}\right)\right\}\right. \\
& \left.+\int_{\theta_{i-1}}^{\theta_{i}}\left[\left(\tilde{p} e_{2}\right)^{\prime \prime}+\left(\tilde{p} \mathrm{e}_{3}\right)^{\prime}\right] f(\theta) \mathrm{d} \theta\right] .
\end{aligned}
$$

By choosing $f$ appropriately, we end up with

$$
\begin{aligned}
& \left(\tilde{p} e_{2}\right)^{\prime \prime}+\left(\tilde{p} e_{3}\right)^{\prime}=0 \quad \text { on }\left(\theta_{i-1}, \theta_{i}\right), i=1, \ldots, n, \\
& \left(\tilde{p} e_{3}+\left(\tilde{p} e_{2}\right)^{\prime}\right)\left(\theta_{i}^{-}\right)=\left(\tilde{p} e_{3}+\left(\tilde{p} e_{2}\right)^{\prime}\right)\left(\theta_{i}^{+}\right), \quad i=1, \ldots, n-1, \\
& \left(\tilde{p} e_{3}+\left(\tilde{p} e_{2}\right)^{\prime}\right)\left(\theta_{0}^{+}\right)=\left(\tilde{p} e_{3}+\left(\tilde{p} e_{2}\right)^{\prime}\right)\left(\theta_{n}^{-}\right), \\
& \left(\tilde{p} e_{2}\right)\left(\theta_{i}^{-}\right)=\left(\tilde{p} e_{2}\right)\left(\theta_{i}^{+}\right), \quad i=1, \ldots, n-1, \\
& \left(\tilde{p} e_{2}\right)\left(\theta_{0}^{+}\right)=\left(\tilde{p} e_{2}\right)\left(\theta_{n}^{-}\right) .
\end{aligned}
$$

Hence by (4.6) on $\left(\theta_{i-1}, \theta_{i}\right), i=1, \ldots, n$ for some $B_{i}$ and $C_{i}$,

$$
\left(\tilde{p} e_{2}\right)(\theta)=h(\theta)\left[C_{i} \int_{0}^{\theta} h(u)^{-1} \mathrm{~d} u+B_{i}\right],
$$


where $h(u)$ is from (4.4). By (4.7) and (4.8)

$$
C_{1}=C_{2}=\cdots=C_{n},
$$

so that

$$
\left(\tilde{p} e_{2}\right)(\theta)=h(\theta)\left[C_{1} \int_{0}^{\theta} h(u)^{-1} \mathrm{~d} u+B_{i}\right] \quad \text { on }\left(\theta_{i-1}, \theta_{i}\right)
$$

Using (4.9),

$$
B_{1}=B_{2}=\cdots=B_{n}
$$

By (4.10),

$$
B_{1}=h(2 \pi)\left[C_{1} \int_{0}^{2 \pi} h(u)^{-1} \mathrm{~d} u+B_{1}\right]
$$

giving

$$
B_{1}[1-h(2 \pi)]=C_{1} h(2 \pi) \int_{0}^{2 \pi} h(u)^{-1} \mathrm{~d} u .
$$

Hence if $h(2 \pi)=1$ then we must have $C_{1}=0$ and so

$$
\left(\tilde{p} e_{2}\right)(\theta)=h(\theta) \mathrm{B}_{1}
$$

which is equivalent to the first part of (4.5).

If $h(2 \pi) \neq 1$, then

$$
B_{1}=C_{1} \frac{h(2 \pi)}{1-h(2 \pi)} \int_{0}^{2 \pi} h(u)^{-1} \mathrm{~d} u
$$

and so

$$
\left(\tilde{p} e_{2}\right)(\theta)=C_{1} h(\theta)\left[\int_{0}^{\theta} h(u)^{-1} \mathrm{~d} u+\frac{h(2 \pi)}{1-h(2 \pi)} \int_{0}^{2 \pi} h(u)^{-1} \mathrm{~d} u\right],
$$

giving the second part of (4.5).

\section{The critical case $\alpha=1$}

In this section we consider a special two-dimensional case. Our main result is the following theorem.

Theorem 5.1. Let $d=2, b_{i}=0$ for $i=1,2$ and suppose $a$ is piecewise constant on $S^{1}$. If $\alpha=1$ and $X_{0} \neq 0$, then $X$ does not hit $\{0\}$ almost surely. Moreover, $X$ hits every 
neighbourhood of $\{0\}$ in finite time almost surely.

Proof. We use the notation from Section 4. In particular, $a$ is constant on each of the intervals $\left(\theta_{i-1}, \theta_{i}\right), i=1, \ldots, n$. Since $\operatorname{tr} a=e_{1}+e_{2}$,

$$
\begin{aligned}
0=\alpha-1 & =\int_{S^{1}} \frac{e_{1}+e_{2}}{e_{1}} p \mathrm{~d} \theta-2 \\
& =\int_{S^{1}}\left[\frac{e_{2}}{e_{1}}-1\right] p \mathrm{~d} \theta .
\end{aligned}
$$

Since $\tilde{p}=p / e_{1}$, this becomes

$$
0=\int_{S^{1}}\left(e_{2}-e_{1}\right) \tilde{p} \mathrm{~d} \theta .
$$

We need the next result, whose proof is deferred to the end of the section.

Theorem 5.2. The condition (5.1) implies the existence of a function $u \in C^{1}\left(S^{1}\right)$ such that for $i=1, \ldots, n$,

$$
\begin{gathered}
u \in C_{b}^{2}\left(\theta_{i-1}, \theta_{i}\right) \\
\bar{L}_{2} u=-\frac{1}{2} \frac{e_{2}-e_{1}}{e_{1}} \quad \text { on }\left(\theta_{i-1}, \theta_{i}\right)
\end{gathered}
$$

Set

$$
f(r, \theta)=\ln r+u(\theta) .
$$

Then for $\theta \in\left(\theta_{i-1}, \theta_{i}\right)$ and $r>0$, by (4.1) and (4.2),

$$
\begin{aligned}
\bar{L} f & =\frac{1}{e_{1}} L f \\
& =\frac{1}{2 r^{2}}\left[\frac{e_{2}-e_{1}}{e_{1}}\right]+\frac{1}{r^{2}} \bar{L}_{2} u \\
& =0 .
\end{aligned}
$$

Since $u \in C_{b}^{2}\left(\theta_{i-1}, \theta_{i}\right) \cap C^{1}\left(S^{1}\right)$, there is an approximating family of functions $f_{n} \in C_{b}^{2}\left(\mathbb{R}^{2}\right)$ such that $f_{n}$ and its first-order partials converge uniformly on $\left\{x \in \mathbb{R}^{2}: \varepsilon<|x|<M\right\}$ to $f$ and its first-order partials, respectively. Moreover, the second-order partials of $f_{n}$ are uniformly bounded on $\left\{x \in \mathbb{R}^{2}: \varepsilon<|x|<M\right\}$, and converge pointwise to the corresponding partials of $f$ on $(\varepsilon, M) \times\left(\theta_{i-1}, \theta_{i}\right)$. Applying the martingale property of $\bar{P}_{x}$ to each $f_{n}$, using optional stopping, and then letting $n \rightarrow \infty$, we obtain that

$$
f\left(X\left(t \wedge \tau_{\varepsilon} \wedge \tau_{M}\right)\right)
$$

is a $\bar{P}_{x}$-martingale. The exceptional set where the second-order partials of $f$ fail to exist is of no consequence because $\bar{P}_{x}$ a.s. $X$ spends zero Lebesgue time there (Krylov 1971). 
Thus, using $\overline{\mathrm{E}}_{x}$ to denote expectation with respect to $\bar{P}_{x}$,

$$
\overline{\mathrm{E}}_{x} f\left(X_{t \wedge \tau_{\varepsilon} \wedge \tau_{M}}\right)=f(x),
$$

and letting $t \rightarrow \infty$,

$$
\overline{\mathrm{E}}_{x} f\left(X_{\tau_{\varepsilon} \wedge \tau_{M}}\right)=f(x) .
$$

Expanding,

$$
(\ln \varepsilon) \bar{P}_{x}\left(\tau_{\varepsilon}<\tau_{M}\right)+(\ln M) \bar{P}_{x}\left(\tau_{\varepsilon}>\tau_{M}\right)+\overline{\mathrm{E}}_{x} u\left(X_{\tau_{\varepsilon} \wedge \tau_{M}}\right)=\ln r+u(\theta) .
$$

Consequently,

$$
\bar{P}_{x}\left(\tau_{\varepsilon}<\tau_{M}\right)=\left[\ln r+u(\theta)-\ln M-\overline{\mathrm{E}}_{x} u\left(X_{\tau_{\varepsilon} \wedge \tau_{M}}\right)\right] /[\ln \varepsilon-\ln M] .
$$

First fix $M$ and let $\varepsilon \rightarrow 0$ to get

$$
\bar{P}_{x}\left(\tau_{0}<\tau_{M}\right)=0, \quad 0<|x|<M,
$$

since $u$ is bounded. Next, fix $\varepsilon$ and let $M \rightarrow \infty$ to get

$$
\bar{P}_{x}\left(\tau_{\varepsilon}<\infty\right)=1, \quad \varepsilon<|x| .
$$

Combined with (5.2), Theorem 5.1 follows.

Proof of Theorem 5.2. We assume $a$ is constant on each of the intervals $\left(\theta_{i-1}, \theta_{i}\right)$, $i=1, \ldots, n$. Then $e_{3}=-e_{2}^{\prime}$ on $\left(\theta_{i-1}, \theta_{i}\right)$ and (4.6)-(4.8) become

$$
\begin{array}{ll}
\left(\tilde{p}^{\prime} e_{2}\right)^{\prime}=0 \text { on }\left(\theta_{i-1}, \theta_{i}\right), & i=1, \ldots, n, \\
\left(\tilde{p}^{\prime} e_{2}\right)\left(\theta_{i}^{-}\right)=\left(\tilde{p}^{\prime} e_{2}\right)\left(\theta_{i}^{+}\right), & i=1, \ldots, n-1 \\
\left(\tilde{p}^{\prime} e_{2}\right)\left(\theta_{0}^{+}\right)=\left(\tilde{p}^{\prime} e_{2}\right)\left(\theta_{n}^{-}\right) . &
\end{array}
$$

In particular, $\tilde{p}^{\prime} e_{2}$ is constant on $S^{1}$, say

$$
\tilde{p}^{\prime} e_{2}(\theta)=\beta \text {. }
$$

Define

$$
H(\theta)=\tilde{p} e_{2}
$$

and observe that, by (4.9) and (4.10), $H$ is continuous, and that, by (4.5), $\inf _{S^{1}} H>0$. Moreover, since $\tilde{p} \in C^{\infty}\left(\theta_{i-1}, \theta_{i}\right)$, so is $H$.

We wish to consider the differential operator

$$
M_{\sigma} u=\left(H u^{\prime}\right)^{\prime}-\beta u^{\prime}-\sigma u
$$

for $\sigma \in \mathbb{R}$. To make sense of this, define

$$
\mathscr{C}_{\sigma}(u, v)=\int_{S^{1}}\left[H u^{\prime} v^{\prime}+\left(\beta u^{\prime}+\sigma u\right) v\right] \mathrm{d} \theta, \quad u, v \in W^{1,2}\left(S^{1}\right) .
$$


For $g \in L^{2}\left(S^{1}\right)$, a weakly differentiable function $u$ is a weak or generalized solution of $M_{\sigma} u=g$ on $S^{1}$ if, for all $v \in C^{1}\left(S^{1}\right)$,

$$
\mathscr{C}_{\sigma}(u, v)=-\int_{S^{1}} g v \mathrm{~d} \theta
$$

Let $M_{\sigma}^{*}$ denote the formal adjoint of $M_{\sigma}$, defined by

$$
M_{\sigma}^{*} u=\left(H u^{\prime}+\beta u\right)^{\prime}-\sigma u .
$$

We use $\mathscr{L}_{\sigma}^{*}$ to denote the corresponding bilinear form:

$$
\mathscr{L}_{\sigma}^{*}(u, v)=\int_{S^{1}}\left[\left(H u^{\prime}+\beta u\right) v^{\prime}+\sigma u v\right] \mathrm{d} \theta, \quad u, v \in W^{1,2}\left(S^{1}\right) .
$$

Clearly

$$
\mathscr{C l}_{\sigma}^{*}(u, v)=\mathscr{C l}_{\sigma}(v, u) .
$$

We interpret the equation $\mathscr{C}_{\sigma}^{*} u=g$ in the weak sense analogous to $M_{\sigma}^{*} u=g$ described above. We can argue much like the proof of Theorem 8.6 in Gilbarg and Trudinger (1983), to end up with the following consequence of the Fredholm alternative in Hilbert space.

Theorem 5.3. There exists a discrete countable set $\sum \subseteq \mathbb{R}$ such that if $\sigma \notin \sum$, the problems

$$
M_{\sigma} u=g, \quad M_{\sigma}^{*} u=g
$$

have unique solutions in $W^{1,2}\left(S^{1}\right)$ for arbitrary $g \in L^{2}\left(S^{1}\right)$. If $\sigma \in \sum$, then the subspaces of solutions of the homogeneous problems

$$
M_{\sigma} u=0, \quad M_{\sigma}^{*} u=0
$$

are of positive, finite dimension and the problem $M_{\sigma} u=g$ is solvable if and only if

$$
\int_{S^{1}} g v \mathrm{~d} \theta=0
$$

for all $v \in W^{1,2}\left(S^{1}\right)$ satisfying $M_{\sigma}^{*} v=0$.

Notice, for $v \in C^{1}\left(S^{1}\right)$, that

$$
\mathscr{C}_{0}^{*}(1, v)=\int_{S^{1}} \beta v^{\prime} \mathrm{d} \theta=0,
$$

since $\beta$ is constant and $v$ is continuous. Hence

$$
M_{0}^{*} 1=0,
$$

and we see that $0 \in \sum$.

Lemma 5.4. Any $v \in W^{1,2}\left(S^{1}\right)$ satisfying $M_{0}^{*} v=0$ must be constant.

Proof. Since $H \in C^{\infty}\left(\theta_{i-1}, \theta_{i}\right), v \in C^{\infty}\left(\theta_{i-1}, \theta_{i}\right)$ for $i=1, \ldots, n$. Hence for $\varphi \in C^{1}\left(S^{1}\right)$, 


$$
\begin{aligned}
0 & =\mathscr{C}_{0}^{*}(v, \varphi)=\sum_{i=1}^{n} \int_{\theta_{i-1}}^{\theta_{i}}\left(H v^{\prime}+\beta v\right) \varphi^{\prime} \mathrm{d} \theta \\
& =\sum_{i=1}^{n}\left[\left(\left[H v^{\prime}+\beta v\right] \varphi\right)\left(\theta_{i}^{-}\right)-\left(\left[H v^{\prime}+\beta v\right] \varphi\right)\left(\theta_{i-1}^{+}\right)-\int_{\theta_{i-1}}^{\theta_{i}}\left(H v^{\prime}+\beta v\right)^{\prime} \varphi \mathrm{d} \theta\right] .
\end{aligned}
$$

By choosing $\varphi$ appropriately, we have

$$
\left(H v^{\prime}+\beta v\right)^{\prime}=0 \quad \text { on }\left(\theta_{i-1}, \theta_{i}\right)
$$

and

$$
\left\{\begin{array}{l}
\left(H v^{\prime}+\beta v\right)\left(\theta_{i}^{-}\right)=\left(H v^{\prime}+\beta v\right)\left(\theta_{i}^{+}\right), \quad i=1, \ldots, n-1 \\
\left(H v^{\prime}+\beta v\right)\left(\theta_{0}^{+}\right)=\left(H v^{\prime}+\beta v\right)\left(\theta_{n}^{-}\right) .
\end{array}\right.
$$

The latter implies $H v^{\prime}+\beta v$ is continuous on $S^{1}$. Hence by (5.9) there is some constant $C_{1}$ such that

$$
H v^{\prime}+\beta v=C_{1} .
$$

If $\beta=0$, then for some constant $C_{2}$,

$$
v(\theta)=\int_{0}^{\theta} C_{1} H(u)^{-1} \mathrm{~d} u+C_{2} .
$$

But $v$ is continuous on $S^{1}$ so we must have $v\left(0^{+}\right)=v\left(2 \pi^{-}\right)$, and this forces $C_{1}=0$ since $H>0$. Thus $v$ is constant.

On the other hand, if $\beta \neq 0$, then for some constant $C_{2}$

$$
v(\theta)=\frac{C_{1}}{\beta}+C_{2} \exp \left\{-\int_{0}^{\theta} \beta H(u)^{-1} \mathrm{~d} u\right\} .
$$

Continuity of $v$ on $S^{1}$ forces $C_{2}=0$ and once again $v$ is constant.

In any case, we have shown $v$ is constant, and the proof of Lemma 5.4 is complete.

Let us apply these results to prove Theorem 5.2. Let

$$
g=-\frac{e_{2}-e_{1}}{e_{2}} .
$$

Then

$$
\begin{aligned}
\int_{S^{1}} H g \mathrm{~d} \theta & =\int_{S^{1}} \tilde{p} e_{2}\left(-\frac{e_{2}-e_{1}}{e_{2}}\right) \mathrm{d} \theta \\
& =-\int_{S^{1}}\left(e_{2}-e_{1}\right) \tilde{p} \mathrm{~d} \theta \\
& =0, \quad \text { by }(5.1) .
\end{aligned}
$$

Hence by Lemma 5.4 and the last part of Theorem 5.3, we have $u \in W^{1,2}\left(S^{1}\right)$ such that 


$$
M_{0} u=H g
$$

Thus, for all $v \in C^{1}\left(S^{1}\right)$,

$$
\begin{aligned}
0 & =\int_{S^{1}}\left[H u^{\prime} v^{\prime}+\beta u^{\prime} v\right] \mathrm{d} \theta+\int_{S^{1}} H g v \mathrm{~d} \theta \\
& =\sum_{i=1}^{n}\left[\left(H u^{\prime} v\right)\left(\theta_{i}^{-}\right)-\left(H u^{\prime} v\right)\left(\theta_{i-1}^{+}\right)-\int_{\theta_{i-1}}^{\theta_{i}}\left[\left(H u^{\prime}\right)^{\prime}-\beta u^{\prime}-H g\right] v \mathrm{~d} \theta\right] .
\end{aligned}
$$

Since $g$ and $H$ are in $C^{\infty}\left(\theta_{i-1}, \theta_{i}\right)$, so is $u$. By choosing $v$ appropriately (5.10) yields

$$
\left(H u^{\prime}\right)^{\prime}-\beta u^{\prime}-H g=0 \quad \text { on }\left(\theta_{i-1}, \theta_{i}\right)
$$

and

$$
\left\{\begin{array}{l}
\left(H u^{\prime}\right)\left(\theta_{i}^{-}\right)=\left(H u^{\prime}\right)\left(\theta_{i}^{+}\right), \quad i=1, \ldots, n-1, \\
\left(H u^{\prime}\right)\left(\theta_{0}^{+}\right)=\left(H u^{\prime}\right)\left(\theta_{n}^{-}\right) .
\end{array}\right.
$$

Then (5.11) becomes

$$
\begin{aligned}
0 & =\left(\tilde{p} e_{2} u^{\prime}\right)^{\prime}-\tilde{p}^{\prime} e_{2} u^{\prime}-\tilde{p} e_{2}\left(-\frac{e_{2}-e_{1}}{e_{2}}\right) \\
& =\tilde{p}\left(e_{2} u^{\prime}\right)^{\prime}+\tilde{p}\left(e_{2}-e_{1}\right) .
\end{aligned}
$$

In particular,

$$
\left(e_{2} u^{\prime}\right)^{\prime}=-\left(e_{2}-e_{1}\right)
$$

Since $e_{3}=-e_{2}^{\prime}$ on $\left(\theta_{i-1}, \theta_{i}\right)$, by (4.2) and (5.13)

$$
\bar{L}_{2} u=-\frac{1}{2}\left(\frac{e_{2}-e_{1}}{e_{1}}\right) \quad \text { on }\left(\theta_{i-1}, \theta_{i}\right) \text {. }
$$

Since $H>0$ is continuous, (5.12) forces $u^{\prime}$ to be continuous.

To finish the proof, we still need to show that $u^{\prime \prime}$ is bounded on $\left(\theta_{i-1}, \theta_{i}\right)$. For this, notice that, on $\left(\theta_{i-1}, \theta_{i}\right)$,

$$
\begin{aligned}
H^{\prime}=\left(\tilde{p} e_{2}\right)^{\prime} & =\tilde{p}^{\prime} e_{2}+\tilde{p} \tilde{e}_{2}^{\prime} \\
& =\beta+\beta e_{2}^{\prime} / e_{2}
\end{aligned}
$$

which is bounded. Hence by (5.11), the boundedness of $g$ and $u^{\prime}$, and inf $H>0$, we have that $u^{\prime \prime}$ is bounded on $\left(\theta_{i-1}, \theta_{i}\right)$, as claimed.

\section{References}

Bass, R.F. and Pardoux, E. (1987) Uniqueness for diffusions with piecewise constant coefficients. Probab. Theory Related Fields, 76, 557-572.

DeBlassie, R.D. (1988) Remark on exit times from cones in $\mathbb{R}^{n}$ of Brownian motion, Probab. Theory 
Related Fields, 79, 95-97.

DeBlassie, R.D. (1998) On hitting single points by a multidimensional diffusion. Stochastics Stochastics Rep., 65, 1-11.

Ethier, S.N. and Kurtz, T.G. (1986) Markov Processes: Characterization and Convergence. New York: Wiley.

Gilbarg, D. and Trudinger, N.S. (1983) Elliptic Partial Differential Equations of Second Order, 2nd edn. Berlin: Springer-Verlag.

Ikeda, N. and Watanabe, S. (1989) Stochastic Differential Equations and Diffusion Processes, 2nd edn. Amsterdam: North-Holland.

Krylov, N.V. (1971) An inequality in the theory of stochastic integrals. Theory Probab. Appl. 16, 438448.

Pinsky, R. (1995) Positive Harmonic Functions and Diffusion Cambridge: Cambridge University Press.

Skorokhod, A.V. (1989) Asymptotic Methods in the Theory of Stochastic Differential Equations. Providence, RI: American Mathematical Society.

Stroock, D.W. and Varadhan, S.R.S. (1979) Multidimensional Diffusion Processes. New York: Springer-Verlag.

Williams, R.J. (1985) Brownian motion with polar drift. Trans. Amer. Math. Soc., 292, 225-246.

Received December 1996 and revised February 1998. 